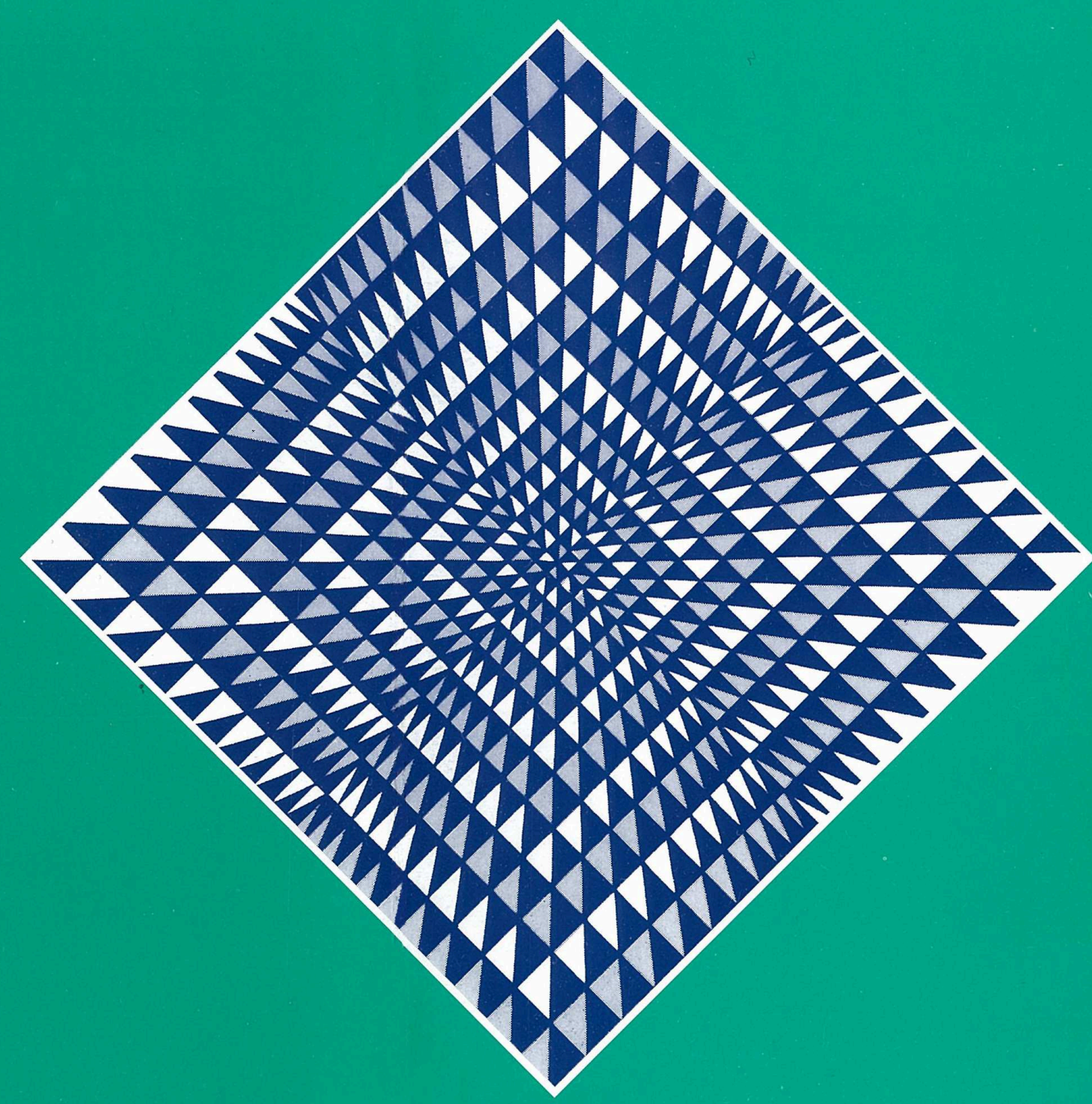

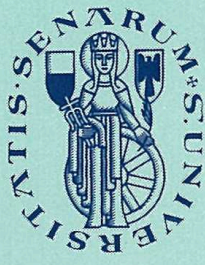

UNIVERSITÀ DEGLI STUDI DI SIENA

DIPARTIMENTO

DI STUDI AZIENDALI

E SOCIALI

Sezione di "Popolazione,

Ambiente e Metodi

Matematico-statistici"

Andrea Menchiari

MULTIPLE DECREMENT

LIFE TABLES

12 


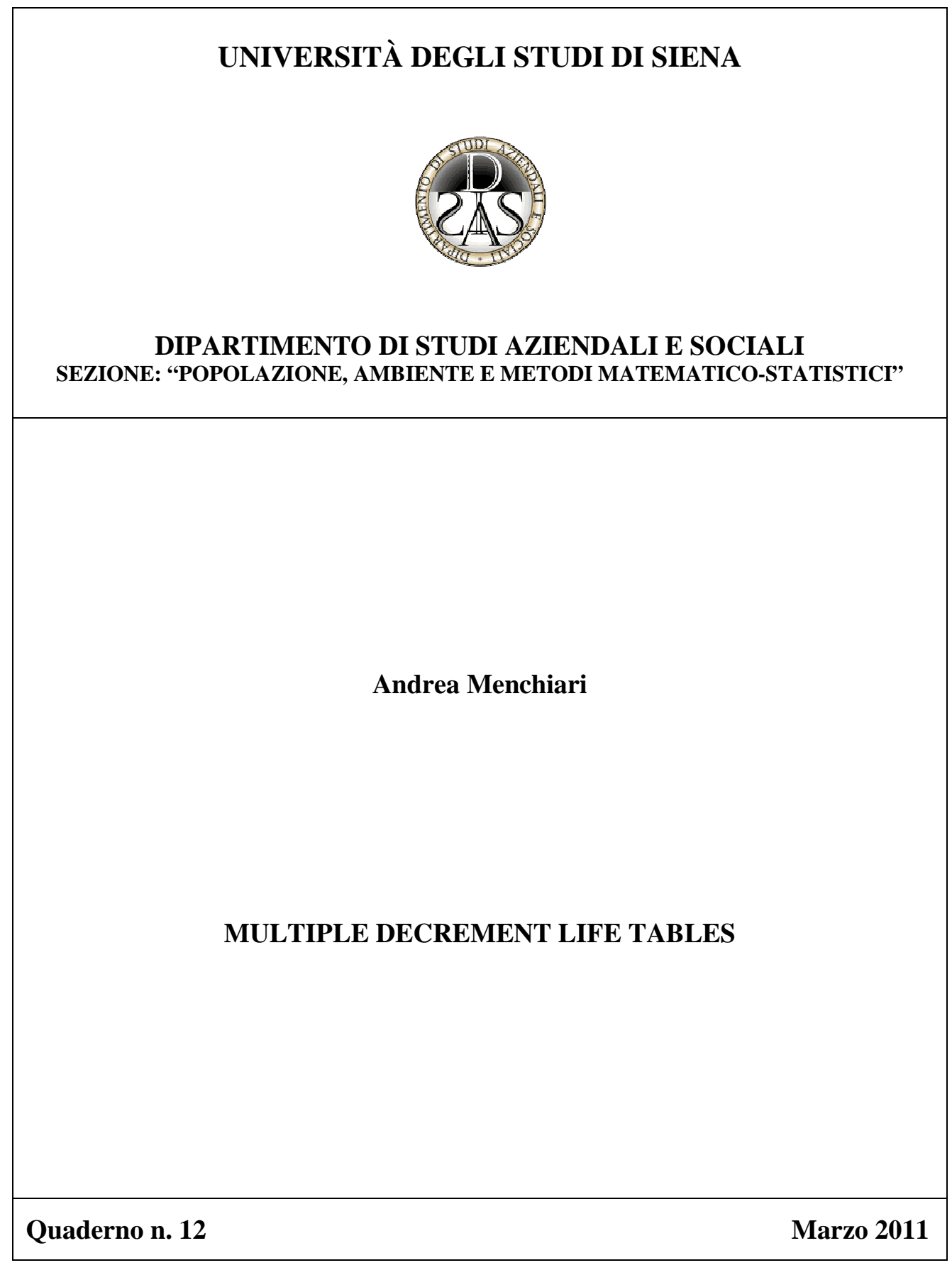


Redazione:

DIPARTIMENTO DI STUDI AZIENDALI E SOCIALI

Università di Siena - Piazza S. Francesco, 8 - 53100 - Siena (Italy)

Telefono: +39 577 232656/232629

Fax: +39577 232641/232629

E-mail: seg.az_soc@unisi.it

Coordinatore:

Franco Fineschi

Comitato di redazione:

Mario Berti

Cinzia Buccianti

Sandra Fontani

ISBN: 978-88-914971-6-6

Proprietà letteraria riservata.

(C) Copyright 2011 - Dipartimento S.A.S. - Università degli Studi di Siena.

A norma della legge sul diritto d'autore e del codice civile è vietata la riproduzione di questo Quaderno o parte di esso con qualsiasi mezzo elettronico o meccanico. Copie dei Quaderni possono comunque essere richieste alla Redazione.

I Quaderni vengono inviati ad Istituti, Dipartimenti, Enti vari, docenti e ricercatori italiani e stranieri che svolgono un'attività scientifica analoga a quella effettuata dalla Sezione che ne cura la pubblicazione. È consentita la partecipazione all'attività dei Quaderni anche a coloro che non fanno parte del Dipartimento di Studi Aziendali e Sociali dell'Università degli Studi di Siena, subordinatamente all'approvazione sia del Comitato di Redazione che di un Referee. 


\title{
MULTIPLE DECREMENT LIFE TABLES
}

\author{
Andrea Menchiari
}

\section{INTRODUZIONE}

Le tavole di "mortalità" o tavole di "sopravvivenza" costituiscono uno strumento fondamentale per l'analisi del processo di eliminazione per età e sesso di una popolazione sottoposta, durante un certo periodo di tempo, ad una serie di eventi di tipo ambientale, economico, sociale, sanitario, ecc.

Il loro campo d'impiego è molto vasto e spazia dall'ambito strettamente demografico, ove vengono utilizzate in un'ampia varietà di casi (confronti multipli tra condizioni di mortalità riscontrate in tempi e/o Paesi diversi, modelli di previsione della popolazione, definizione di popolazioni stabili e semi-stabili, ecc.), all' ambito attuariale, ove risultano indispensabili per operazioni quali il calcolo del premio assicurativo in caso di morte, delle rendite vitalizie, ecc.

Ulteriori applicazioni riguardano sia il campo biologico, dove vengono impiegate per studiare il processo di eliminazione con l'età di specie animali e vegetali, sia il controllo statistico di qualità, dove il processo di eliminazione riguarda degli oggetti sottoposti ad un utilizzo controllato che, con il passare del tempo, ne provoca prima l'usura e poi la rottura.

Ogni tavola di sopravvivenza è composta da una serie di funzioni biometriche, ognuna delle quali ha una sua peculiarità e consente di analizzare un particolare aspetto del processo di eliminazione per età e sesso al quale è sottoposta una data popolazione.

Così vi troviamo i quozienti di mortalità, che esprimono il rischio di morte all'interno di un dato intervallo di età, i sopravviventi, che partendo da un contingente iniziale (radice della tavola) vengono via via eliminati, la distribuzione dei decessi, la speranza di vita, ossia il numero di anni che mediamente restano da vivere a persone che hanno già raggiunto una data età, la resistenza alla mortalità, ed altre funzioni ancora.

In particolare, se ci concentriamo sui decessi che si manifestano con il trascorrere dell'età, ognuno di questi può essere interpretato come un "passaggio di stato", ossia un movimento di un individuo dallo stato di 
"vivo" allo stato di "deceduto". Questo movimento (che può avvenire solo in un senso), prende il nome di "decremento" e comporta una progressiva eliminazione della popolazione iniziale man mano che l'età aumenta e si avvicina ad un valore estremo $\omega$ (in genere: $110 \leq \omega \leq 120$ anni), in corrispondenza del quale nessun individuo è più in vita.

Tavole di mortalità di questo tipo, che prevedono un unico passaggio dallo stato di "vivo" a quello di "deceduto", sono indicate con la notazione di tavole a "decrementi singoli".

Una situazione più complessa si ha quando non ci limitiamo a considerare il solo stato di "deceduto", ma consideriamo anche la causa per la quale è avvenuto il decesso. In questo caso, se supponiamo di avere $k$ cause di morte $(1,2,3, \ldots, i, \ldots, k)$, ogni individuo passerà dallo stato di "vivo" a quello di "deceduto per la causa $i$-esima", configurando così una tavola di mortalità a "decrementi multipli".

\section{DIPENDENZA TRA CAUSE DI MORTE}

In una tavola di mortalità a "decrementi multipli" l'ipotesi di base è che le cause di decesso siano mutualmente esclusive; ossia, se una persona muore per una data causa non può morire anche per un'altra. In questa situazione le varie cause, rappresentano dei "competing risks" ai quali è sottoposto ogni individuo in funzione della propria età, ma alla fine tra queste soltanto una sarà la causa alla quale sarà imputabile un decesso.

Sotto tali condizioni, risulta evidente che le varie cause di morte sono tra di loro "dipendenti", in quanto l'aumentare (o il diminuire) dell'incidenza di una causa, a parità di tutte le altre condizioni, comporterà il diminuire (o l'aumentare) dell'incidenza delle altre cause di morte.

In sostanza, se diminuisce l'incidenza delle morti per tumore, le persone che non moriranno più per questa causa andranno ad aumentare il numero di persone morte per altre cause e, conseguentemente, varieranno anche le relative probabilità di decesso.

Ai fini della nostra ricerca, volendo costruire delle tavole di mortalità a "decrementi multipli", possiamo semplificare lo schema di calcolo senza perdere in generalità, considerando due sole cause di morte: la i-esima e l'insieme delle restanti cause diverse dalla i-esima. 
Se ${ }_{n} d_{x}$ sono i decessi rilevati in una tavola di mortalità, nell'intervallo di età $[x ; x+n]$ (ossia tra i compleanni: $x$ incluso e $x+n$ escluso), mentre ${ }_{n} d_{x ; i} \mathrm{e}_{n} d_{x ; \neq i}$ sono i decessi imputabili, rispettivamente, alla causa di morte i-esima e diversa dalla $i$-esima, per quanto precedentemente enunciato, sarà:

$$
{ }_{n} d_{x}={ }_{n} d_{x ; i}+{ }_{n} d_{x ; \neq i}
$$

Per la stima dei decessi per causa di morte, generalmente viene fatta l'ipotesi che questi seguano la stessa distribuzione per età dei decessi osservati nella popolazione reale, con riferimento allo stesso intervallo di tempo utilizzato per la costruzione della tavola a "decrementi singoli". Quindi, indicati con ${ }_{n} D_{x},{ }_{n} D_{x ; i} \mathrm{e}_{n} D_{x ; \neq i}$ tali decessi, avremo:

$$
\begin{aligned}
& { }_{n} D_{x}={ }_{n} D_{x ; i}+{ }_{n} D_{x ; \neq i} \\
& { }_{n} d_{x ; i} \cong \frac{{ }_{n} D_{x ; i}}{{ }_{n} D_{x}} \cdot{ }_{n} d_{x} \\
& { }_{n} d_{x ; \neq i} \cong \frac{{ }_{n} D_{x ; \neq i}}{{ }_{n} D_{x}} \cdot{ }_{n} d_{x}
\end{aligned}
$$

Indicati con $l_{x} \mathrm{i}$ sopravviventi all'età esatta $x$, della tavola di mortalità a "decrementi singoli" e con ${ }_{n} q_{x}$ la probabilità di decesso nell'intervallo $[x ; x+n]$ per individui che hanno raggiunto l'età $x$, è possibile ottenere le corrispondenti probabilità di decesso per causa di morte, nell'ipotesi di "dipendenza" tra le cause:

$$
\begin{aligned}
& { }_{n} \bar{q}_{x ; i}=\frac{{ }_{n} d_{x ; i}}{l_{x}} \cong \frac{\frac{{ }_{n} D_{x ; i}}{{ }_{n} D_{x}} \cdot{ }_{n} d_{x}}{l_{x}} \cong \frac{{ }_{n} d_{x}}{l_{x}} \cdot \frac{{ }_{n} D_{x ; i}}{{ }_{n} D_{x}} \cong{ }_{n} q_{x} \cdot \frac{{ }_{n} D_{x ; i}}{{ }_{n} D_{x}} \\
& { }_{n} \bar{q}_{x ; \neq i}=\frac{{ }_{n} d_{x ; \neq i}}{l_{x}} \cong \frac{\frac{{ }_{n} D_{x ; \neq i}}{{ }_{n} D_{x}} \cdot{ }_{n} d_{x}}{l_{x}} \cong \frac{{ }_{n} d_{x}}{l_{x}} \cdot \frac{{ }_{n} D_{x ; \neq i}}{{ }_{n} D_{x}} \cong{ }_{n} q_{x} \cdot \frac{{ }_{n} D_{x ; \neq i}}{{ }_{n} D_{x}}
\end{aligned}
$$


Dalle relazioni precedenti, inoltre, ne consegue che:

$$
{ }_{n} q_{x}={ }_{n} q_{x} \cdot \frac{{ }_{n} D_{x ; i}}{{ }_{n} D_{x}}+{ }_{n} q_{x} \cdot \frac{{ }_{n} D_{x ; \neq i}}{{ }_{n} D_{x}}={ }_{n} \bar{q}_{x ; i}+{ }_{n} \bar{q}_{x ; \neq i}
$$

ossia, la probabilità che un individuo in vita all'età esatta $x$ muoia prima di aver raggiunto l'età $x+n$ si può ottenere sommando le corrispondenti probabilità per causa di morte, qualora queste risultino tra di loro "dipendenti”.

\section{INDIPENDENZA TRA CAUSE DI MORTE}

Consideriamo un'ipotetica popolazione sottoposta a $k$ possibili cause di morte $(1,2,3, \ldots, i, \ldots, k)$, tra di loro "indipendenti". In tali condizioni, la probabilità di decesso per la causa i-esima indica il rischio di morte al quale è sottoposto ogni individuo, nell'ipotesi che la i-esima causa di morte sia l'unica possibile.

La differenza con il caso precedente è evidente. Sotto l'ipotesi di "dipendenza" tra cause di morte, infatti, la stessa probabilità di decesso per la causa i-esima indica il rischio di morte al quale è sottoposto ogni individuo in presenza di tutte le altre cause, che agiscono contemporaneamente e che costituiscono dei "competing risks".

In altre parole, nel caso di "indipendenza" tra cause di morte, diversamente dalla condizione di "dipendenza", la probabilità di decesso per la causa $i$ esima non risente di variazioni, anche consistenti, delle probabilità di decesso per le altre cause, che ai fini pratici è come se non esistessero.

Per distinguere i due casi, si fa spesso riferimento a probabilità di morte "grezze" (in caso di "dipendenza") e "nette" (in caso di "indipendenza").

Come è stato fatto in precedenza, lo schema di calcolo può essere semplificato senza perdere di generalità, considerando due sole cause di morte tra di loro "indipendenti": la i-esima e l'insieme delle restanti cause diverse dalla $i$-esima.

Sulla base di quanto sopra possiamo indicare con $q_{i}(x)$ la probabilità che un individuo appartenente al contingente iniziale radice della tavola, muoia 
prima di aver raggiunto l'età $x$ per la causa i-esima (in assenza di tutte le altre cause diverse dalla $i$-esima $)$ e con $q_{\neq i}(x)$ la probabilità che muoia per una causa diversa dalla $i$-esima (in assenza della causa $i$-esima).

Essendo $q_{i}(x)$ e $q_{\neq i}(x)$ le probabilità di decesso, allora, $p_{i}(x)=1-q_{i}(x)$ e $p_{\neq i}(x)=1-q_{\neq i}(x)$ saranno le corrispondenti probabilità di sopravvivenza, dalla nascita sino all'età $x$, per un individuo sottoposto al solo rischio di morte $i$ esimo o soltanto all'insieme dei rischi di morte diversi dall' $i$-esimo.

Nell'ipotesi di "indipendenza" tra cause di morte, potremo scrivere:

$$
p(x)=p_{i}(x) \cdot p_{\neq i}(x)
$$

ossia, la probabilità di sopravvivenza dalla nascita all'età $x$ può essere scomposta nel prodotto tra le corrispondenti probabilità di sopravvivenza per la causa $i$-esima e per le restanti cause diverse dalla $i$-esima.

Dalla (3.1), calcolando il logaritmo di ambo i membri e derivando, si ottiene:

$$
\frac{d}{d x}\{\ln [p(x)]\}=\frac{d}{d x}\left\{\ln \left[p_{i}(x)\right]\right\}+\frac{d}{d x}\left\{\ln \left[p_{\neq i}(x)\right]\right\}
$$

da cui:

$\left\{\frac{d}{d x}[p(x)]\right\} \cdot \frac{1}{p(x)}=\left\{\frac{d}{d x}\left[p_{i}(x)\right]\right\} \cdot \frac{1}{p_{i}(x)}+\left\{\frac{d}{d x}\left[p_{\neq i}(x)\right]\right\} \cdot \frac{1}{p_{\neq i}(x)}$

la quale, cambiando di segno, essendo: $\mu(x)=-p^{\prime}(x) / p(x)$, può essere scritta:

$$
\mu(x)=\mu_{i}(x)+\mu_{\neq i}(x)
$$

ossia, il tasso istantaneo di mortalità $\mu(x)$ o "forza di mortalità", se le cause di morte sono tra loro "indipendenti", può essere scomposto, ad ogni età $x$, nella somma dei tassi istantanei per causa di morte.

Supponiamo, adesso, di voler calcolare la probabilità di decesso tra le età $x \mathrm{e}$ $x+n$ per la $i$-esima causa di morte, nell'ipotesi di "indipendenza" tra cause. A tale scopo, facendo riferimento alla simbologia sin qui adottata, possiamo 
inizialmente utilizzare la relazione esistente che lega la probabilità di sopravvivenza al corrispondente tasso istantaneo di mortalità:

$$
\frac{p_{i}(x+n)}{p_{i}(x)}=e^{-\int_{x}^{x+n} \mu_{i}(z) \cdot d z}
$$

dalla quale, calcolando il logaritmo di ambo i membri, quindi dividendo e moltiplicando per una stessa quantità, si ottiene:

$$
\ln \left[\frac{p_{i}(x+n)}{p_{i}(x)}\right]=-\frac{\int_{x}^{x+n} \mu_{i}(z) \cdot d z}{\int_{x}^{x+n} \mu(z) \cdot d z} \cdot \int_{x}^{x+n} \mu(z) \cdot d z
$$

Quest'ultima relazione può essere ulteriormente sviluppata. Analogamente alla (3.5), infatti, potremo scrivere:

$$
\frac{p(x+n)}{p(x)}=e^{-\int_{x}^{x+n} \mu(z) \cdot d z}
$$

dalla quale, calcolando il logaritmo di ambo i membri, si ottiene:

$$
-\int_{x}^{x+n} \mu(z) \cdot d z=\ln \left[\frac{p(x+n)}{p(x)}\right]
$$

Sostituendo questo termine in quello più a destra nella (3.6) si ha:

$$
\ln \left[\frac{p_{i}(x+n)}{p_{i}(x)}\right]=\frac{\int_{x}^{x+n} \mu_{i}(z) \cdot d z}{\int_{x}^{x+n} \mu(z) \cdot d z} \cdot \ln \left[\frac{p(x+n)}{p(x)}\right]
$$

A questo punto, se introduciamo l'ipotesi semplificatrice che i tassi istantanei di mortalità siano costanti all'interno dell'intervallo di età $[x ; x+n]$, ossia: $\mu(x)=\mu$ e $\mu_{i}(x)=\mu_{i}$, avremo: 
$\ln \left[\frac{p_{i}(x+n)}{p_{i}(x)}\right] \cong \frac{\mu_{i}}{\mu} \cdot \ln \left[\frac{p(x+n)}{p(x)}\right]$

che può anche essere scritta:

$\left[\frac{p_{i}(x+n)}{p_{i}(x)}\right] \cong\left[\frac{p(x+n)}{p(x)}\right]^{\left(\frac{\mu_{i}}{\mu}\right)}$

Inoltre, essendo: ${ }_{n} p_{x ; i}=p_{i}(x+n) / p_{i}(x)$ e ${ }_{n} p_{x}=p(x+n) / p(x)$ le probabilità di sopravvivenza tra le età $x$ e $x+n$, rispettivamente, per la causa $i$-esima e per tutte le cause, avremo:

${ }_{n} p_{x ; i} \cong{ }_{n} p_{x}\left(\frac{\mu_{i}}{\mu}\right)$

la quale, a sua volta, per la relazione esistente tra probabilità di sopravvivenza e probabilità di morte, ossia: ${ }_{n} p_{x ; i}=1{ }_{n} q_{x ; i}$ e ${ }_{n} p_{x}=1{ }_{n} q_{x}$, può essere scritta:

${ }_{n} q_{x ; i} \cong 1-\left(1-{ }_{n} q_{x}\right)^{\left(\frac{\mu_{i}}{\mu}\right)}$

Facendo l'ulteriore ipotesi semplificatrice che il tasso istantaneo di mortalità, che abbiamo supposto costante all'interno dell'intervallo di età $[x ; x+n]$, possa essere approssimato dal corrispondente tasso specifico di mortalità calcolato sulla popolazione reale, ossia: $\mu_{i} \simeq_{n} D_{x ; i} /{ }_{n} P_{x}$ e $\mu \cong{ }_{n} D_{x}{ }_{n} P_{x}$, dove ${ }_{n} D_{x ; i}$ e ${ }_{n} D_{x}$ sono, rispettivamente, i decessi osservati nell'intervallo di età $[x ; x+n]$ per la causa i-esima e per tutte le cause, mentre ${ }_{n} P_{x}$ è la popolazione media osservata nello stesso intervallo di età, abbiamo:

${ }_{n} q_{x ; i} \cong 1-\left(1-{ }_{n} q_{x}\right)^{\left(\frac{{ }_{n} D_{x ; i}}{{ }_{n} D_{x}}\right)}$ 
Analogamente, possiamo calcolare la probabilità che un individuo in vita al compleanno $x$-esimo, muoia prima di compiere $x+n$ anni, nell'ipotesi che in tale intervallo di età sia sottoposto al rischio di morte soltanto per cause diverse dall' $i$-esima e che quest'ultima causa non abbia alcuna rilevanza, ossia:

${ }_{n} q_{x ; \neq i} \cong 1-\left(1-{ }_{n} q_{x}\right)\left(\frac{{ }_{n} D_{x ; \neq i}}{{ }_{n} D_{x}}\right)$

Infine, utilizzando le relazioni precedentemente esposte, è facile dimostrare che:

${ }_{n} q_{x}={ }_{n} q_{x ; i}+{ }_{n} q_{x ; \neq i}-{ }_{n} q_{x ; i} \cdot{ }_{n} q_{x ; \neq i}$

mediante la quale è possibile scomporre la probabilità di decesso nell'intervallo di età $[x ; x+n]$, utilizzando le corrispondenti probabilità di decesso per la causa di morte i-esima e per tutte le altre cause diverse dalla i-esima, nell'ipotesi di "indipendenza" tra cause precedentemente enunciata.

\section{RELAZIONE TRA QUOZIENTI “DIPENDENTI” ED “INDIPENDENTI”}

Nei capitoli precedenti sono state analizzate due diverse metodologie per ottenere tavole di sopravvivenza a "decrementi multipli", in presenza di una serie di cause di morte, ipotizzate tra loro "dipendenti" oppure "indipendenti".

I valori dei quozienti ottenuti con i due procedimenti, in genere, non coincidono. Per ogni classe di età e per ogni causa di morte, infatti, i valori dei quozienti ottenuti sotto l'ipotesi di "indipendenza" sono maggiori dei corrispondenti valori ottenuti nel caso di "dipendenza". La sola eccezione si ha quando la probabilità di decesso in una certa classe di età risulta nulla; in questo caso anche la probabilità di morte per una qualsiasi causa, sia questa “dipendente" o "indipendente", sarà nulla. 
Tra i quozienti calcolati nel caso di "dipendenza" o di "indipendenza" è possibile stabilire delle relazioni ben precise. A questo proposito, dalla (2.5) si ricava facilmente:

$\frac{{ }_{n} D_{x ; i}}{{ }_{n} D_{x}}=\frac{{ }_{n} \bar{q}_{x ; i}}{{ }_{n} q_{x}}$

la quale, sostituita nella (3.14), consente di ottenere:

$$
{ }_{n} q_{x ; i}=1-\left(1-{ }_{n} q_{x}\right)^{\left(\frac{{ }_{n} \bar{q}_{x ; i}}{{ }_{n} q_{x}}\right)}
$$

Analogamente, impiegando la (2.6) e la (3.15), si ha:

$$
{ }_{n} q_{x ; \neq i}=1-\left(1-{ }_{n} q_{x}\right)^{\left(\frac{{ }_{n} \bar{q}_{x ; \neq i}}{{ }_{n} q_{x}}\right)}
$$

Dalla (4.2) e (4.3), inoltre, si ottiene:

$$
\begin{aligned}
& { }_{n} \bar{q}_{x ; i}=\left[\frac{\ln \left(1-{ }_{n} q_{x ; i}\right)}{\ln \left(1-{ }_{n} q_{x}\right)}\right] \cdot{ }_{n} q_{x} \\
& { }_{n} \bar{q}_{x ; \neq i}=\left[\frac{\ln \left(1-{ }_{n} q_{x ; \neq i}\right)}{\ln \left(1-{ }_{n} q_{x}\right)}\right] \cdot{ }_{n} q_{x}
\end{aligned}
$$

Queste relazioni consentono il passaggio tra quozienti "dipendenti" e "indipendenti" (o viceversa), anche se il loro impiego è subordinato alla disponibilità delle probabilità di morte ${ }_{n} q_{x}$ della tavola a "decrementi singoli”.

Alternativamente, è possibile impiegare delle relazioni approssimate che richiedono la sola conoscenza dei quozienti "dipendenti” o "indipendenti", 
senza far ricorso alla probabilità di decesso ${ }_{n} q_{x}$. A tal proposito Hinde (1998) ha dimostrato che:

$$
\begin{aligned}
& { }_{n} \bar{q}_{x ; i} \cong{ }_{n} q_{x ; i}\left(1-\frac{1}{2} \cdot{ }_{n} q_{x ; \neq i}\right) \\
& { }_{n} \bar{q}_{x ; \neq i} \cong{ }_{n} q_{x ; \neq i}\left(1-\frac{1}{2} \cdot{ }_{n} q_{x ; i}\right)
\end{aligned}
$$

dalle quali, calcolando le relazioni inverse:

$$
\begin{aligned}
& { }_{n} q_{x ; i} \cong \frac{{ }_{n} \bar{q}_{x ; i}}{\left(1-\frac{1}{2} \cdot{ }_{n} q_{x ; \neq i}\right)} \\
& { }_{n} q_{x ; \neq i} \cong \frac{{ }_{n} \bar{q}_{x ; \neq i}}{\left(1-\frac{1}{2} \cdot{ }_{n} q_{x ; i}\right)}
\end{aligned}
$$

In questo ultimo caso, per risolvere simultaneamente la (4.8) e la (4.9), occorre far ricorso ad un procedimento iterativo, usando i quozienti "dipendenti" come valori di partenza per i corrispondenti quozienti "indipendenti" e quindi sostituendo questi ultimi con i valori ottenuti risolvendo le relazioni precedenti. In genere, la convergenza richiede poche iterazioni e risulta estremamente rapida.

Alternativamente, è possibile fermarsi alla prima iterazione, accontentandosi di una peggiore approssimazione, impiegando le relazioni:

$$
{ }_{n} q_{x ; i} \cong \frac{{ }_{n} \bar{q}_{x ; i}}{\left(1-\frac{1}{2} \cdot{ }_{n} \bar{q}_{x ; \neq i}\right)}
$$




$$
{ }_{n} q_{x ; \neq i} \cong \frac{{ }_{n} \bar{q}_{x ; \neq i}}{\left(1-\frac{1}{2} \cdot{ }_{n} \bar{q}_{x ; i}\right)}
$$

Nei casi esaminati, a parità di tutte le altre condizioni, le approssimazioni risulteranno tanto migliori quanto più piccole in valore assoluto risulteranno le probabilità di decesso nei vari intervalli di età.

\section{TAVOLE DI MORTALITÀ A “DECREMENTI MULTIPLI" - ITALIA - 2007}

Utilizzando la metodologia esposta nei precedenti capitoli sono state costruite delle tavole di sopravvivenza a "decrementi multipli", nell'ipotesi che alcune cause di morte vengano completamente eliminate. Come dati di base sono state impiegate le tavole di mortalità della popolazione italiana, relative all'anno 2007, pubblicate dall'ISTAT separatamente per i due sessi, nonché le distribuzioni dei decessi per sesso e causa di morte, calcolate per la popolazione italiana nello stesso anno.

In particolare, dalla classificazione internazionale "ridotta" ICD 10, che prevede diciassette diverse cause di morte, sono state selezionate le tre più rilevanti per la popolazione italiana maschile e femminile nell'anno 2007, ossia: le "malattie del sistema circolatorio", i "tumori" e le "malattie del sistema respiratorio". Per completare l'analisi, un ulteriore gruppo di cause di morte è stato considerato, ossia: le "cause esterne di traumatismo e avvelenamento"; questo gruppo, come incidenza, è risultato al quarto posto per la popolazione maschile ed al settimo per la popolazione femminile.

L'elevata incidenza delle tre principali cause di morte esaminate rappresenta il punto di arrivo di un lungo processo evolutivo della mortalità italiana, processo che si è sviluppato per oltre un secolo nel corso del quale, oltre ad una riduzione generalizzata dei rischi di morte, si è assistito ad una radicale redistribuzione dei decessi per causa.

Pur tenendo presenti le probabili carenze ed imprecisioni che agli inizi del secolo scorso potevano caratterizzare le rilevazioni sulle cause di morte e la loro non sufficiente comparabilità con i dati più recenti, dovuta anche al susseguirsi di sostanziali revisioni delle prime classificazioni, risulta 
Tabella 1 - Distribuzione dei decessi secondo le cause di morte - Italia - 2007

\begin{tabular}{|c|c|c|c|c|c|}
\hline \hline $\begin{array}{c}\text { European } \\
\text { Short List } \\
\text { ICD10 }\end{array}$ & $\begin{array}{c}\text { Cause } \\
\text { di } \\
\text { morte }\end{array}$ & $\begin{array}{c}\text { Deceduti } \\
\text { Maschi } \\
\text { Val. assoluti }\end{array}$ & $\begin{array}{c}\text { Deceduti } \\
\text { Maschi } \\
\text { Valori \% }\end{array}$ & $\begin{array}{c}\text { Deceduti } \\
\text { Femmine } \\
\text { Val. assoluti }\end{array}$ & $\begin{array}{c}\text { Deceduti } \\
\text { Femmine } \\
\text { Valori \% }\end{array}$ \\
\hline I00-I99 & $\begin{array}{c}\text { Malattie del } \\
\text { sistema } \\
\text { circolatorio }\end{array}$ & 97366 & 34,67 & 126945 & 43.47 \\
\hline C00-D48 & Tumori & 97355 & 34.66 & 74270 & 25.43 \\
\hline J00-J99 & $\begin{array}{c}\text { Malattie del } \\
\text { sistema } \\
\text { respiratorio }\end{array}$ & 21389 & 7.62 & 16423 & 5.62 \\
\hline V01-Y89 & $\begin{array}{c}\text { Cause esterne di } \\
\text { traumatismo e } \\
\text { avvelenamento }\end{array}$ & 14732 & 5.25 & 9727 & 3.33 \\
\hline ---- & $\begin{array}{c}\text { Altre cause } \\
\text { diverse dalle } \\
\text { precedenti }\end{array}$ & 50009 & 17.80 & 64665 & 22.15 \\
\hline \hline Totale & \begin{tabular}{c} 
Tutte le cause \\
\hline
\end{tabular} & 280851 & 100.00 & 292030 & 100,00 \\
\hline
\end{tabular}

evidente che alcune malattie (in particolare quelle di natura infettiva) hanno progressivamente ridotto la loro incidenza lasciando il posto ad altre malattie di tipo cronico degenerativo (quali tumori e malattie del sistema circolatorio), delle quali risultano affette, soprattutto, le persone in età avanzata.

Se, infatti, oggi alle "malattie del sistema circolatorio" ed ai "tumori" è imputabile circa il 70\% dei decessi sia per i maschi che per le femmine, agli inizi del 1900 l'incidenza di tali cause era minima (circa il 10\%), mentre assai rilevanti erano cause di decesso come: le "malattie dell'apparato respiratorio", le "malattie dell'apparato digerente" e le "malattie infettive e parassitarie", che oggi, nel complesso, hanno un'incidenza piuttosto limitata (di poco superiore al $13 \%$ per i maschi ed al $10 \%$ per le femmine). 
In sostanza, i progressi raggiunti in campo medico-farmacologico, insieme al miglioramento delle condizioni di vita, hanno permesso, da un lato, di ridurre notevolmente il grado di letalità di numerose malattie, favorendo l'allungamento della vita e, dall'altro, il proliferare di patologie di tipo degenerativo, tipiche di persone che, essendo in età avanzata, oppongono una minor resistenza alla mortalità.

Tornando alle tavole di mortalità a "decrementi multipli", queste sono state costruite, separatamente per i due sessi, rimuovendo di volta in volta una delle cause di morte precedentemente indicate.

Le tavole di sopravvivenza così ottenute, confrontate con la corrispondente tavola maschile o femminile a "decrementi singoli" (Italia, 1970), hanno permesso di valutare quale sarebbe stato l'impatto sulla longevità umana dell'eliminazione di una data causa di morte.

A questo proposito, per ogni intervallo di età e separatamente per i due sessi, sono stati confrontati i quozienti di mortalità osservati ${ }_{n} q_{x}$ con i corrispondenti quozienti ${ }_{n} q_{x ; \neq i}$ ottenuti impiegando la (3.15). In particolare, è stata calcolata la differenza ${ }_{n} q_{x}-{ }_{n} q_{x ; \neq i}$, ossia la riduzione della probabilità di morte nella classe di età $[x ; x+n]$ per le persone che hanno già raggiunto l'età $x$, nel caso in cui la i-esima causa di morte venisse completamente eliminata

Analogamente, sempre operando per età e sesso, è stata calcolata la differenza tra la speranza di vita $e_{x ; \neq i}$ della tavola a "decrementi multipli" e la corrispondente speranza di vita $e_{x}$ della tavola a "decrementi singoli", ossia: $e_{x ; \neq i}-e_{x}$. Tale differenza ha permesso di valutare, ad una certa età $x$, quale sarebbe stato il guadagno nella speranza di vita in assenza della $i$ esima causa di morte.

I risultati completi sono riportati nelle Tabelle 1-8 dell'APPENDICE, mentre una sintesi è esposta qui di seguito:

5.1 MAlATTIE DEL SISTEMA CIRCOLATORIO - Le malattie del sistema circolatorio costituiscono la prima causa di morte per la popolazione italiana. I decessi dovuti a tale causa, infatti, hanno rappresentato nel 2007 il 34,67\% della mortalità maschile ed il 43,47\% di quella femminile (Tabella 1). L'eliminazione di questa causa porterebbe ad una sostanziale riduzione delle probabilità di morte, soprattutto nelle età avanzate (80-100 anni), con un abbattimento di tali valori di oltre il 30\% 
per i maschi e di oltre il $40 \%$ per le femmine. L'eliminazione totale delle malattie del sistema circolatorio avrebbe anche, come diretta conseguenza, l'innalzamento della speranza di vita alla nascita di 4,12 anni per i maschi e di 4,72 anni per le femmine. L'incremento della speranza di vita risulterebbe rilevante anche alle età avanzate; all'età di 90 anni, per esempio, si avrebbe ancora un incremento di 3,03 anni per i maschi e di 3,77 per le femmine. Nelle Figure 1-2 e 3-4 sono riportati gli andamenti dei sopravviventi e delle probabilità di morte (in scala logaritmica), separatamente per i maschi e per le femmine.

5.2 TUMORI - I tumori rappresentano la seconda causa di morte per la popolazione italiana (Tabella 1); la loro incidenza nel 2007 è risultata maggiore per i maschi $(34,66 \%$ del totale dei decessi per questo sesso) rispetto alle femmine $(25,43 \%)$. Stante ciò, l'ipotetica eliminazione dei tumori come causa di morte è evidente che avvantaggerebbe soprattutto il sesso maschile. L'incremento della vita media alla nascita, nel caso si realizzasse questa ipotesi, sarebbe di 4,28 anni per i maschi e di 3,27 per le femmine. A differenza del caso precedente gli incrementi della speranza di vita a seguito dell'eliminazione dei tumori come causa di morte decrescono abbastanza velocemente dopo i 60 anni, tanto che all'età di 90 anni l'incremento atteso della speranza di vita è di soli 0,87 anni per i maschi e 0,63 per le femmine. Per quanto riguarda invece l'abbattimento delle probabilità di morte, per i maschi questo risulterebbe di oltre il 30\% (con punte anche del 51\%) nell'intervallo di età 45-80 anni, mentre, per le femmine si avrebbe una riduzione delle probabilità di morte di oltre il 30\% (con punte del 64\%) nell'intervallo di età 25-80 anni. Da notare che, anche nell'intervallo di età 5-10 anni, si avrebbe una riduzione delle probabilità di morte di oltre il 38\% sia per i maschi che per le femmine.

5.3 MALATTIE DEL SISTEMA RESPIRATORIO - Le malattie del sistema respiratorio costituiscono la terza causa di morte della popolazione italiana (Tabella 1). Rispetto alle due cause precedentemente esaminate la loro incidenza è molto più bassa: 7,62\% per i maschi e 5,62\% per le femmine. L'eliminazione di questa causa porterebbe ad una lieve riduzione delle probabilità di morte su tutto l'intervallo di età. La maggiore riduzione riguarderebbe la classe di età 80-85 anni, per la 

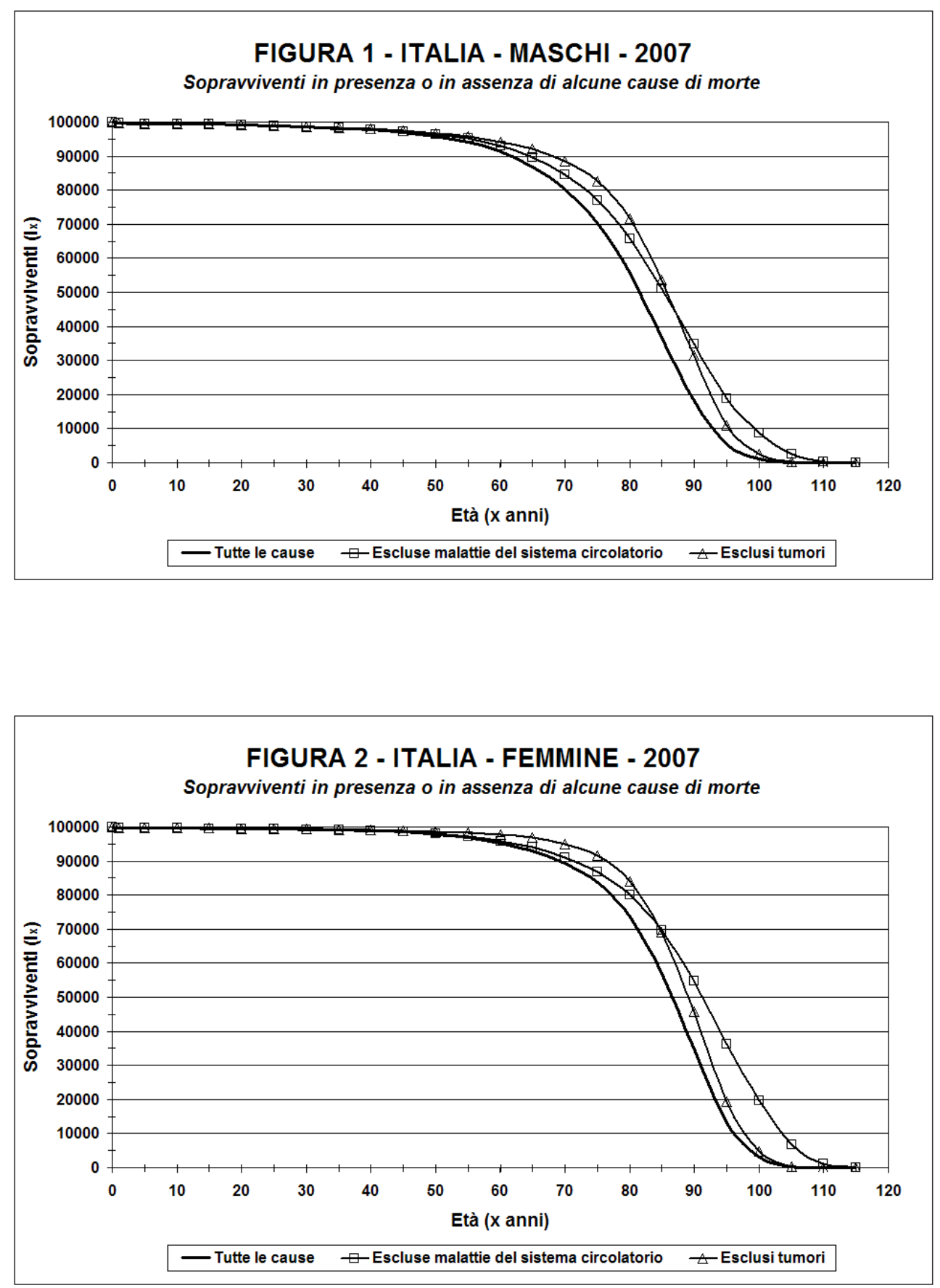

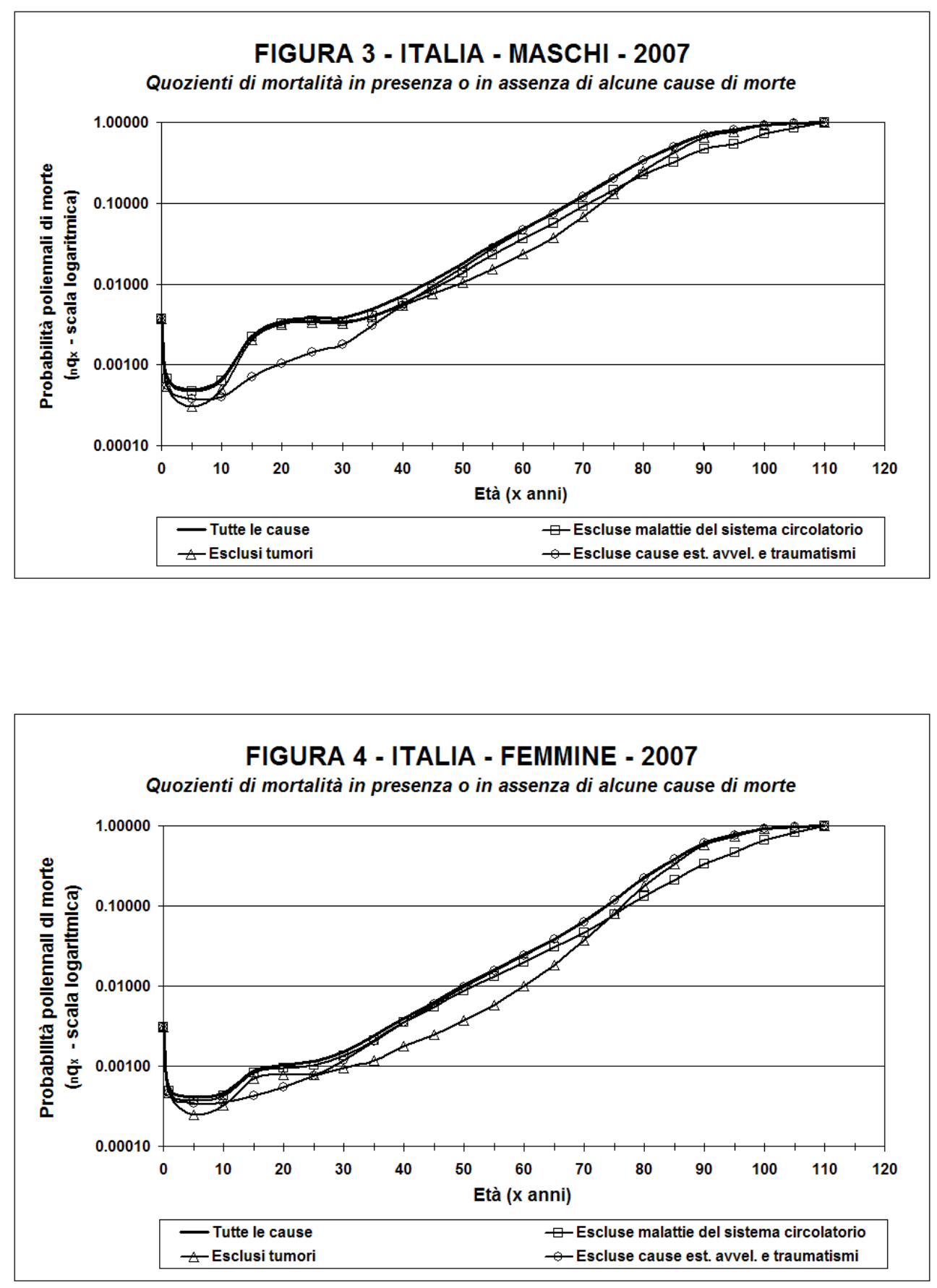
quale si avrebbe un abbattimento delle probabilità di morte dell' $8 \%$ per i maschi e del $5 \%$ per le femmine. Anche i guadagni nella speranza di vita sarebbero lievi e sempre inferiori all'anno, indipendentemente dall' età e dal sesso.

5.4 CAUSE ESTERNE DI TRAUMATISMO ED AVVELENAMENTO - I traumatismi e gli avvelenamenti costituiscono la quarta causa di morte $(5,25 \%)$ per la popolazione maschile italiana e la settima $(3,33 \%)$ per la popolazione femminile (Tabella 1). Essendo la popolazione maschile più soggetta a decessi per questa causa ne consegue che la sua ipotetica eliminazione porterebbe un incremento della speranza di vita alla nascita più elevato per i maschi (1,02 anni) rispetto alle femmine (soltanto 0,41 anni). Le fasce d'età all'interno delle quali si avrebbero le maggiori riduzioni delle probabilità di morte sarebbero 10-40 anni per i maschi e 15-30 anni per le femmine. All'interno di questi intervalli di età, avremmo un abbattimento delle probabilità di morte sempre superiore al $30 \%$, con punte del $69,52 \%$ per i maschie e del $50,83 \%$ per le femmine. Occorre, tuttavia, notare che tali riduzioni, pur essendo cospicue in termini percentuali, risultano poco consistenti in termini assoluti, in quanto le probabilità di morte in tali intervalli di età risultano essere estremamente basse. Le implicazioni dell'eliminazione di questa causa di morte possono essere osservate graficamente nelle Figure 3 e 4, nelle quali per mettere in rilievo quanto sopra esposto, le probabilità di morte sono riportate in scala logaritmica. 


\section{APPENDICE}

\section{TAVOLE DI MORTALITÀ DELLA POPOLAZIONE ITALIANA PER ETÀ E SESSO (IN PRESENZA OD IN ASSENZA DI ALCUNE CAUSE DI MORTE)}

Nel corso del nostro lavoro ci siamo limitati a considerare le quattro cause di morte $(i=1,2,3,4)$ più significative per la popolazione italiana, ossia:

1) Malattie del sistema circolatorio.

2) Tumori.

3) Malattie del sistema respiratorio.

4) Cause esterne di traumatismo ed avvelenamento.

Il significato e le modalità di calcolo delle funzioni biometriche presenti nelle tavole in APPENDICE sono i seguenti:

Colonna $\mathbf{A}-[x]$ - Età espressa in anni. Le tavole di mortalità sono riportate in forma "abbreviata" $(x=0,1,5,10,15, \ldots, 95,100)$.

Colonna B $-\left[{ }_{n} D_{x}\right]$ - Numero di decessi osservati per la popolazione italiana (maschile o femminile) nell'anno 2007, indipendentemente dalla causa che ha provocato la morte.

Colonna C - $\left.{ }_{n} D_{x ; i}\right]$ - Numero di decessi osservati per la popolazione italiana (maschile o femminile) nell'anno 2007, imputabili alla causa di morte $i$-esima.

Colonna D - $\left.{ }_{n} D_{x ; \neq i}={ }_{n} D_{x^{-} n} D_{x ; i}\right]$ - Numero di decessi osservati per la popolazione italiana (maschile o femminile) nell'anno 2007, dovuti ad una qualsiasi causa di morte purché diversa dalla i-esima.

Colonna E - $\left.{ }_{n} q_{x}\right]$ - Quoziente di mortalità o probabilità di morte. nell'intervallo di età $[x ; x+n]$. Esprime il rischio che una persona di età $x$ muoia prima di aver raggiunto l'età $x+n$, indipendentemente dalla causa del decesso. 
Colonna $\mathbf{F}-\left[{ }_{n} q_{x ; \neq i}\right]$ - Quoziente di mortalità o probabilità di morte nell'intervallo di età $[x ; x+n]$, calcolato nell'ipotesi in cui la causa $i$ esima sia stata rimossa. Esprime il rischio che una persona di età $x$ muoia prima di aver raggiunto l'età $x+n$, per una causa di morte diversa dall' $i$-esima.

Colonna G - $\left.{ }_{n} q_{x^{-} n} q_{x ; \neq i}\right]$ - Esprime, per ogni intervallo di età $[x ; x+n]$, la riduzione assoluta del rischio di morte, al quale sono sottoposti i viventi in età $x$, a seguito dell' eliminazione della causa $i$ esima.

Colonna H $-\left[100 \cdot\left({ }_{n} q_{x^{-} n} q_{x ; \neq i}\right){ }_{n} q_{x}\right]$ - Esprime, per ogni intervallo di età $[x ; x+n]$, la riduzione percentuale del rischio di morte, al quale sono sottoposti i viventi in età $x$, nel caso in cui la causa di morte $i$ esima venisse eliminata.

Colonna I $-\left[e_{x}\right]-$ Vita media o speranza di vita all'età $x$. Esprime il numero di anni che mediamente restano da vivere ad una persona che ha già raggiunto l'età $x$, qualora agiscano tutte le cause di morte.

Colonna $\mathbf{L}-\left[e_{x ; \neq i}\right]$ - Vita media o speranza di vita all'età $x$, calcolata nel caso in cui la $i$-esima causa di morte sia stata eliminata. Esprime il numero di anni che mediamente restano da vivere ad una persona che ha già raggiunto l'età $x$, qualora agiscano tutte le cause di morte tranne la i-esima.

Colonna $\mathbf{M}-\left[e_{x ; \neq i}-e_{x}\right]$ - Esprime l'incremento assoluto della speranza di vita all'età $x$, nel caso in cui la $i$-esima causa di morte venga rimossa.

Colonna $\mathbf{N}-\left[100 \cdot\left(e_{x ; \neq i}-e_{x}\right) / e_{x}\right]-$ Esprime l'incremento percentuale della speranza di vita all'età $x$, nel caso in cui la i-esima causa di morte venga rimossa. 
TABELLA 1 - Tavole di mortalità in presenza ed in assenza della causa di morte: "Malattie del sistema circolatorio" - Italia - Maschi - 2007.

\begin{tabular}{|c|c|c|c|c|c|c|c|c|c|c|c|}
\hline \multirow[b]{2}{*}{ Età $x$} & \multicolumn{3}{|c|}{ Decessi osservati } & \multicolumn{4}{|c|}{ Quozienti di mortalità } & \multicolumn{4}{|c|}{ Speranza di vita } \\
\hline & ${ }_{n} D_{x}$ & ${ }_{n} D_{x, i}$ & ${ }_{n} D_{x, \neq i}$ & $n q_{x}$ & ${ }_{n} q_{x, \neq i}$ & Diff. ass. & Diff. \% & $\mathbf{e}_{\mathrm{x}}$ & $\mathbf{e}_{\mathbf{x}, \neq i}$ & Diff. ass. & Diff. \% \\
\hline A & B & C & $D=B-C$ & E & $\mathbf{F}$ & $G=E-F$ & $H=100^{*} G / E$ & I & $\mathbf{L}$ & $M=L-I$ & $\mathrm{~N}=100 * \mathrm{M} / \mathrm{I}$ \\
\hline 0 & 1070 & 25 & 1045 & 0.00379 & 0.00370 & 0.00009 & 2.33 & 78.67 & 82.78 & 4.12 & 5.23 \\
\hline 1 & 197 & 10 & 187 & 0.00070 & 0.00067 & 0.00004 & 5.07 & 77.97 & 82.09 & 4.12 & 5.29 \\
\hline 5 & 153 & 10 & 143 & 0.00050 & 0.00047 & 0.00003 & 6.53 & 74.02 & 78.15 & 4.12 & 5.57 \\
\hline 10 & 198 & 12 & 186 & 0.00067 & 0.00063 & 0.00004 & 6.06 & 69.06 & 73.18 & 4.12 & 5.97 \\
\hline 15 & 759 & 27 & 732 & 0.00227 & 0.00219 & 0.00008 & 3.55 & 64.10 & 68.23 & 4.12 & 6.43 \\
\hline 20 & 1153 & 62 & 1091 & 0.00343 & 0.00324 & 0.00018 & 5.37 & 59.24 & 63.37 & 4.13 & 6.97 \\
\hline 25 & 1473 & 106 & 1367 & 0.00378 & 0.00351 & 0.00027 & 7.18 & 54.44 & 58.57 & 4.13 & 7.59 \\
\hline 30 & 1736 & 176 & 1560 & 0.00383 & 0.00344 & 0.00039 & 10.12 & 49.63 & 53.77 & 4.13 & 8.32 \\
\hline 35 & 2469 & 394 & 2075 & 0.00482 & 0.00405 & 0.00077 & 15.93 & 44.82 & 48.94 & 4.13 & 9.21 \\
\hline 40 & 3629 & 707 & 2922 & 0.00714 & 0.00575 & 0.00139 & 19.43 & 40.02 & 44.13 & 4.11 & 10.28 \\
\hline 45 & 4995 & 1162 & 3833 & 0.01110 & 0.00853 & 0.00257 & 23.16 & 35.29 & 39.37 & 4.08 & 11.57 \\
\hline 50 & 6988 & 1728 & 5260 & 0.01803 & 0.01361 & 0.00443 & 24.56 & 30.65 & 34.69 & 4.04 & 13.16 \\
\hline 55 & 11075 & 2711 & 8364 & 0.02995 & 0.02271 & 0.00725 & 24.20 & 26.17 & 30.13 & 3.97 & 15.15 \\
\hline 60 & 16021 & 3958 & 12062 & 0.04818 & 0.03650 & 0.01168 & 24.25 & 21.89 & 25.78 & 3.88 & 17.73 \\
\hline 65 & 24320 & 6343 & 17977 & 0.07582 & 0.05661 & 0.01920 & 25.33 & 17.87 & 21.66 & 3.79 & 21.23 \\
\hline 70 & 33993 & 9802 & 24191 & 0.12462 & 0.09037 & 0.03425 & 27.48 & 14.12 & 17.81 & 3.69 & 26.16 \\
\hline 75 & 47018 & 15714 & 31304 & 0.20759 & 0.14351 & 0.06408 & 30.87 & 10.74 & 14.33 & 3.58 & 33.37 \\
\hline 80 & 53986 & 21347 & 32639 & 0.34063 & 0.22260 & 0.11804 & 34.65 & 7.87 & 11.31 & 3.44 & 43.73 \\
\hline 85 & 38495 & 17192 & 21304 & 0.50408 & 0.32168 & 0.18240 & 36.19 & 5.61 & 8.83 & 3.22 & 57.44 \\
\hline 90 & 22888 & 11478 & 11409 & 0.71110 & 0.46150 & 0.24960 & 35.10 & 3.81 & 6.83 & 3.03 & 79.51 \\
\hline 95 & 7469 & 3994 & 3475 & 0.80946 & 0.53760 & 0.27186 & 33.59 & 2.95 & 5.55 & 2.60 & 88.08 \\
\hline 100 & 765 & 407 & 358 & 0.93420 & 0.72019 & 0.21401 & 22.91 & 2.04 & 4.10 & 2.06 & 101.09 \\
\hline
\end{tabular}


TABELLA 2 - Tavole di mortalità in presenza ed in assenza della causa di morte: "Malattie del sistema circolatorio" - Italia - Femmine - 2007.

\begin{tabular}{|c|c|c|c|c|c|c|c|c|c|c|c|}
\hline \multirow[b]{2}{*}{ Età $x$} & \multicolumn{3}{|c|}{ Decessi osservati } & \multicolumn{4}{|c|}{ Quozienti di mortalità } & \multicolumn{4}{|c|}{ Speranza di vita } \\
\hline & ${ }_{n} D_{x}$ & ${ }_{n} D_{x, i}$ & ${ }_{n} D_{x, \neq i}$ & $n q_{x}$ & ${ }_{n} q_{x, \neq i}$ & Diff. ass. & Diff. \% & $\mathbf{e}_{\mathrm{x}}$ & $\mathbf{e}_{\mathbf{x}, \neq i}$ & Diff. ass. & Diff. \% \\
\hline A & B & C & $D=B-C$ & E & $\mathbf{F}$ & $G=E-F$ & $H=100^{*} G / E$ & I & $\mathbf{L}$ & $M=L-I$ & $\mathrm{~N}=100 * \mathrm{M} / \mathrm{I}$ \\
\hline 0 & 889 & 15 & 874 & 0.00314 & 0.00309 & 0.00005 & 1.68 & 84.04 & 88.76 & 4.72 & 5.61 \\
\hline 1 & 164 & 18 & 146 & 0.00055 & 0.00049 & 0.00006 & 10.97 & 83.31 & 88.04 & 4.73 & 5.68 \\
\hline 5 & 113 & 12 & 101 & 0.00041 & 0.00037 & 0.00004 & 10.62 & 79.35 & 84.08 & 4.73 & 5.96 \\
\hline 10 & 139 & 11 & 128 & 0.00046 & 0.00043 & 0.00004 & 7.91 & 74.38 & 79.11 & 4.72 & 6.35 \\
\hline 15 & 299 & 16 & 283 & 0.00086 & 0.00082 & 0.00005 & 5.35 & 69.42 & 74.14 & 4.72 & 6.80 \\
\hline 20 & 316 & 25 & 291 & 0.00102 & 0.00094 & 0.00008 & 7.91 & 64.47 & 69.20 & 4.72 & 7.33 \\
\hline 25 & 423 & 44 & 379 & 0.00114 & 0.00102 & 0.00012 & 10.40 & 59.54 & 64.26 & 4.72 & 7.93 \\
\hline 30 & 656 & 81 & 575 & 0.00152 & 0.00133 & 0.00019 & 12.34 & 54.60 & 59.32 & 4.72 & 8.65 \\
\hline 35 & 1155 & 138 & 1017 & 0.00238 & 0.00210 & 0.00028 & 11.94 & 49.68 & 54.40 & 4.72 & 9.50 \\
\hline 40 & 1970 & 215 & 1755 & 0.00398 & 0.00354 & 0.00043 & 10.89 & 44.79 & 49.51 & 4.72 & 10.53 \\
\hline 45 & 2864 & 367 & 2497 & 0.00633 & 0.00552 & 0.00081 & 12.78 & 39.96 & 44.68 & 4.71 & 11.80 \\
\hline 50 & 4066 & 573 & 3493 & 0.01016 & 0.00873 & 0.00143 & 14.03 & 35.20 & 39.91 & 4.71 & 13.39 \\
\hline 55 & 6142 & 943 & 5199 & 0.01573 & 0.01333 & 0.00240 & 15.25 & 30.53 & 35.24 & 4.71 & 15.42 \\
\hline 60 & 8628 & 1567 & 7061 & 0.02450 & 0.02009 & 0.00440 & 17.98 & 25.98 & 30.68 & 4.70 & 18.11 \\
\hline 65 & 13873 & 3019 & 10854 & 0.03876 & 0.03045 & 0.00830 & 21.43 & 21.56 & 26.26 & 4.70 & 21.79 \\
\hline 70 & 20980 & 6165 & 14815 & 0.06465 & 0.04610 & 0.01855 & 28.70 & 17.32 & 22.01 & 4.68 & 27.02 \\
\hline 75 & 36275 & 13199 & 23076 & 0.11945 & 0.07774 & 0.04172 & 34.92 & 13.33 & 17.95 & 4.61 & 34.61 \\
\hline 80 & 57518 & 25777 & 31740 & 0.22631 & 0.13203 & 0.09429 & 41.66 & 9.77 & 14.25 & 4.48 & 45.79 \\
\hline 85 & 58471 & 30252 & 28218 & 0.38820 & 0.21111 & 0.17709 & 45.62 & 6.85 & 11.04 & 4.19 & 61.17 \\
\hline 90 & 49734 & 28466 & 21267 & 0.61885 & 0.33798 & 0.28086 & 45.38 & 4.56 & 8.32 & 3.77 & 82.71 \\
\hline 95 & 23434 & 13772 & 9662 & 0.77428 & 0.45866 & 0.31562 & 40.76 & 3.23 & 6.30 & 3.07 & 95.05 \\
\hline 100 & 3921 & 2268 & 1653 & 0.92195 & 0.65877 & 0.26318 & 28.55 & 2.15 & 4.51 & 2.36 & 109.77 \\
\hline
\end{tabular}


TABELLA 3 - Tavole di mortalità in presenza ed in assenza della causa di morte: "Tumori" - Italia Maschi - 2007.

\begin{tabular}{|c|c|c|c|c|c|c|c|c|c|c|c|}
\hline \multirow[b]{2}{*}{ Età $x$} & \multicolumn{3}{|c|}{ Decessi osservati } & \multicolumn{4}{|c|}{ Quozienti di mortalità } & \multicolumn{4}{|c|}{ Speranza di vita } \\
\hline & ${ }_{n} D_{x}$ & ${ }_{n} D_{x, i}$ & ${ }_{n} D_{x, \neq i}$ & $n q_{x}$ & $n q_{x, \neq i}$ & Diff. ass. & Diff. \% & $\mathbf{e}_{\mathrm{x}}$ & $\mathbf{e}_{\mathbf{x}, \neq i}$ & Diff. ass. & Diff. $\%$ \\
\hline A & B & C & $D=B-C$ & E & $\mathbf{F}$ & $G=E-F$ & $H=100^{\star} G / E$ & I & $\mathbf{L}$ & $M=L-I$ & $N=100 * M / I$ \\
\hline 0 & 1070 & 9 & 1061 & 0.00379 & 0.00376 & 0.00003 & 0.84 & 78.67 & 82.95 & 4.28 & 5.44 \\
\hline 1 & 197 & 45 & 152 & 0.00070 & 0.00054 & 0.00016 & 22.83 & 77.97 & 82.26 & 4.29 & 5.50 \\
\hline 5 & 153 & 59 & 94 & 0.00050 & 0.00031 & 0.00019 & 38.54 & 74.02 & 78.30 & 4.28 & 5.78 \\
\hline 10 & 198 & 51 & 147 & 0.00067 & 0.00050 & 0.00017 & 25.74 & 69.06 & 73.33 & 4.27 & 6.18 \\
\hline 15 & 759 & 70 & 689 & 0.00227 & 0.00206 & 0.00021 & 9.22 & 64.10 & 68.36 & 4.26 & 6.64 \\
\hline 20 & 1153 & 107 & 1046 & 0.00343 & 0.00311 & 0.00032 & 9.26 & 59.24 & 63.50 & 4.26 & 7.18 \\
\hline 25 & 1473 & 167 & 1306 & 0.00378 & 0.00335 & 0.00043 & 11.31 & 54.44 & 58.69 & 4.25 & 7.81 \\
\hline 30 & 1736 & 263 & 1473 & 0.00383 & 0.00325 & 0.00058 & 15.13 & 49.63 & 53.88 & 4.24 & 8.55 \\
\hline 35 & 2469 & 456 & 2013 & 0.00482 & 0.00393 & 0.00089 & 18.44 & 44.82 & 49.04 & 4.23 & 9.44 \\
\hline 40 & 3629 & 860 & 2769 & 0.00714 & 0.00545 & 0.00169 & 23.64 & 40.02 & 44.23 & 4.21 & 10.52 \\
\hline 45 & 4995 & 1652 & 3343 & 0.01110 & 0.00744 & 0.00366 & 32.95 & 35.29 & 39.46 & 4.17 & 11.81 \\
\hline 50 & 6988 & 3004 & 3984 & 0.01803 & 0.01032 & 0.00771 & 42.76 & 30.65 & 34.73 & 4.08 & 13.31 \\
\hline 55 & 11075 & 5484 & 5591 & 0.02995 & 0.01524 & 0.01472 & 49.14 & 26.17 & 30.07 & 3.90 & 14.91 \\
\hline 60 & 16021 & 8314 & 7706 & 0.04818 & 0.02347 & 0.02471 & 51.28 & 21.89 & 25.50 & 3.60 & 16.46 \\
\hline 65 & 24320 & 12495 & 11825 & 0.07582 & 0.03761 & 0.03821 & 50.39 & 17.87 & 21.05 & 3.19 & 17.83 \\
\hline 70 & 33993 & 15890 & 18103 & 0.12462 & 0.06843 & 0.05619 & 45.09 & 14.12 & 16.78 & 2.66 & 18.84 \\
\hline 75 & 47018 & 18560 & 28458 & 0.20759 & 0.13136 & 0.07623 & 36.72 & 10.74 & 12.82 & 2.08 & 19.36 \\
\hline 80 & 53986 & 16557 & 37429 & 0.34063 & 0.25079 & 0.08984 & 26.37 & 7.87 & 9.38 & 1.52 & 19.27 \\
\hline 85 & 38495 & 8930 & 29565 & 0.50408 & 0.41646 & 0.08762 & 17.38 & 5.61 & 6.69 & 1.08 & 19.24 \\
\hline 90 & 22888 & 3523 & 19365 & 0.71110 & 0.65026 & 0.06085 & 8.56 & 3.81 & 4.68 & 0.87 & 22.90 \\
\hline 95 & 7469 & 801 & 6668 & 0.80946 & 0.77238 & 0.03708 & 4.58 & 2.95 & 3.73 & 0.78 & 26.45 \\
\hline 100 & 765 & 56 & 709 & 0.93420 & 0.91970 & 0.01450 & 1.55 & 2.04 & 2.91 & 0.87 & 42.85 \\
\hline
\end{tabular}


TABELLA 4 - Tavole di mortalità in presenza ed in assenza della causa di morte: "Tumori" - Italia Femmine - 2007.

\begin{tabular}{|c|c|c|c|c|c|c|c|c|c|c|c|}
\hline \multirow[b]{2}{*}{ Età $x$} & \multicolumn{3}{|c|}{ Decessi osservati } & \multicolumn{4}{|c|}{ Quozienti di mortalità } & \multicolumn{4}{|c|}{ Speranza di vita } \\
\hline & ${ }_{n} D_{x}$ & ${ }_{n} D_{x, i}$ & ${ }_{n} D_{x, \neq i}$ & $n q_{x}$ & $n q_{x, \neq i}$ & Diff. ass. & Diff. \% & $\mathbf{e}_{\mathrm{x}}$ & $\mathbf{e}_{\mathbf{x}, \neq i}$ & Diff. ass. & Diff. $\%$ \\
\hline A & B & C & $D=B-C$ & E & $\mathbf{F}$ & $G=E-F$ & $H=100^{*} G / E$ & I & $\mathbf{L}$ & $M=L-I$ & $N=100 * M / I$ \\
\hline 0 & 889 & 9 & 880 & 0.00314 & 0.00311 & 0.00003 & 1.01 & 84.04 & 87.31 & 3.27 & 3.89 \\
\hline 1 & 164 & 25 & 139 & 0.00055 & 0.00047 & 0.00008 & 15.24 & 83.31 & 86.58 & 3.28 & 3.93 \\
\hline 5 & 113 & 45 & 68 & 0.00041 & 0.00025 & 0.00016 & 39.82 & 79.35 & 82.62 & 3.27 & 4.12 \\
\hline 10 & 139 & 40 & 99 & 0.00046 & 0.00033 & 0.00013 & 28.77 & 74.38 & 77.64 & 3.26 & 4.38 \\
\hline 15 & 299 & 58 & 241 & 0.00086 & 0.00070 & 0.00017 & 19.39 & 69.42 & 72.67 & 3.25 & 4.68 \\
\hline 20 & 316 & 71 & 245 & 0.00102 & 0.00079 & 0.00023 & 22.46 & 64.47 & 67.71 & 3.24 & 5.03 \\
\hline 25 & 423 & 133 & 290 & 0.00114 & 0.00078 & 0.00036 & 31.43 & 59.54 & 62.77 & 3.23 & 5.42 \\
\hline 30 & 656 & 252 & 404 & 0.00152 & 0.00094 & 0.00058 & 38.40 & 54.60 & 57.81 & 3.21 & 5.88 \\
\hline 35 & 1155 & 582 & 573 & 0.00238 & 0.00118 & 0.00120 & 50.36 & 49.68 & 52.87 & 3.18 & 6.41 \\
\hline 40 & 1970 & 1101 & 869 & 0.00398 & 0.00176 & 0.00222 & 55.84 & 44.79 & 47.92 & 3.13 & 6.99 \\
\hline 45 & 2864 & 1751 & 1113 & 0.00633 & 0.00246 & 0.00386 & 61.06 & 39.96 & 43.00 & 3.04 & 7.62 \\
\hline 50 & 4066 & 2585 & 1481 & 0.01016 & 0.00371 & 0.00645 & 63.46 & 35.20 & 38.10 & 2.91 & 8.26 \\
\hline 55 & 6142 & 3913 & 2229 & 0.01573 & 0.00574 & 0.00999 & 63.53 & 30.53 & 33.24 & 2.70 & 8.86 \\
\hline 60 & 8628 & 5099 & 3529 & 0.02450 & 0.01009 & 0.01440 & 58.80 & 25.98 & 28.41 & 2.44 & 9.38 \\
\hline 65 & 13873 & 7457 & 6416 & 0.03876 & 0.01812 & 0.02064 & 53.26 & 21.56 & 23.68 & 2.12 & 9.82 \\
\hline 70 & 20980 & 9073 & 11907 & 0.06465 & 0.03722 & 0.02743 & 42.43 & 17.32 & 19.07 & 1.75 & 10.07 \\
\hline 75 & 36275 & 12060 & 24215 & 0.11945 & 0.08141 & 0.03804 & 31.84 & 13.33 & 14.71 & 1.38 & 10.32 \\
\hline 80 & 57518 & 13458 & 44060 & 0.22631 & 0.17844 & 0.04787 & 21.15 & 9.77 & 10.79 & 1.02 & 10.41 \\
\hline 85 & 58471 & 9575 & 48896 & 0.38820 & 0.33694 & 0.05126 & 13.20 & 6.85 & 7.59 & 0.74 & 10.87 \\
\hline 90 & 49734 & 5180 & 44554 & 0.61885 & 0.57857 & 0.04028 & 6.51 & 4.56 & 5.18 & 0.63 & 13.74 \\
\hline 95 & 23434 & 1640 & 21794 & 0.77428 & 0.74950 & 0.02478 & 3.20 & 3.23 & 3.86 & 0.64 & 19.68 \\
\hline 100 & 3921 & 163 & 3758 & 0.92195 & 0.91322 & 0.00873 & 0.95 & 2.15 & 2.94 & 0.79 & 36.78 \\
\hline
\end{tabular}


TABELLA 5 - Tavole di mortalità in presenza ed in assenza della causa di morte: "Malattie del sistema respiratorio" - Italia - Maschi - 2007.

\begin{tabular}{|c|c|c|c|c|c|c|c|c|c|c|c|}
\hline \multirow[b]{2}{*}{ Età $x$} & \multicolumn{3}{|c|}{ Decessi osservati } & \multicolumn{4}{|c|}{ Quozienti di mortalità } & \multicolumn{4}{|c|}{ Speranza di vita } \\
\hline & ${ }_{n} D_{x}$ & ${ }_{n} D_{x, i}$ & ${ }_{n} D_{x, \neq i}$ & $n q_{x}$ & $n q_{x, \neq i}$ & Diff. ass. & Diff. \% & $\mathbf{e}_{\mathrm{x}}$ & $\mathbf{e}_{x, \neq i}$ & Diff. ass. & Diff. \% \\
\hline A & B & C & $D=B-C$ & E & $\mathbf{F}$ & $G=E-F$ & $H=100^{*} G / E$ & $\mathbf{I}$ & $\mathbf{L}$ & $M=L-I$ & $\mathrm{~N}=100^{\star} \mathrm{M} / \mathrm{I}$ \\
\hline 0 & 1070 & 15 & 1055 & 0.00379 & 0.00374 & 0.00005 & 1.40 & 78.67 & 79.31 & 0.64 & 0.82 \\
\hline 1 & 197 & 9 & 188 & 0.00070 & 0.00067 & 0.00003 & 4.57 & 77.97 & 78.61 & 0.64 & 0.82 \\
\hline 5 & 153 & 5 & 148 & 0.00050 & 0.00049 & 0.00002 & 3.27 & 74.02 & 74.66 & 0.64 & 0.86 \\
\hline 10 & 198 & 3 & 195 & 0.00067 & 0.00066 & 0.00001 & 1.51 & 69.06 & 69.70 & 0.64 & 0.92 \\
\hline 15 & 759 & 10 & 749 & 0.00227 & 0.00224 & 0.00003 & 1.32 & 64.10 & 64.74 & 0.64 & 1.00 \\
\hline 20 & 1153 & 12 & 1141 & 0.00343 & 0.00339 & 0.00004 & 1.04 & 59.24 & 59.88 & 0.64 & 1.08 \\
\hline 25 & 1473 & 8 & 1465 & 0.00378 & 0.00376 & 0.00002 & 0.54 & 54.44 & 55.08 & 0.64 & 1.17 \\
\hline 30 & 1736 & 26 & 1710 & 0.00383 & 0.00377 & 0.00006 & 1.50 & 49.63 & 50.28 & 0.64 & 1.29 \\
\hline 35 & 2469 & 50 & 2419 & 0.00482 & 0.00472 & 0.00010 & 2.02 & 44.82 & 45.46 & 0.64 & 1.43 \\
\hline 40 & 3629 & 97 & 3532 & 0.00714 & 0.00695 & 0.00019 & 2.66 & 40.02 & 40.66 & 0.64 & 1.60 \\
\hline 45 & 4995 & 102 & 4893 & 0.01110 & 0.01087 & 0.00023 & 2.03 & 35.29 & 35.93 & 0.64 & 1.81 \\
\hline 50 & 6988 & 155 & 6833 & 0.01803 & 0.01764 & 0.00040 & 2.20 & 30.65 & 31.29 & 0.64 & 2.09 \\
\hline 55 & 11075 & 286 & 10789 & 0.02995 & 0.02919 & 0.00076 & 2.54 & 26.17 & 26.81 & 0.64 & 2.46 \\
\hline 60 & 16021 & 532 & 15489 & 0.04818 & 0.04662 & 0.00156 & 3.24 & 21.89 & 22.54 & 0.65 & 2.96 \\
\hline 65 & 24320 & 994 & 23326 & 0.07582 & 0.07283 & 0.00298 & 3.93 & 17.87 & 18.52 & 0.66 & 3.68 \\
\hline 70 & 33993 & 1974 & 32019 & 0.12462 & 0.11783 & 0.00679 & 5.45 & 14.12 & 14.78 & 0.67 & 4.72 \\
\hline 75 & 47018 & 3696 & 43322 & 0.20759 & 0.19296 & 0.01463 & 7.05 & 10.74 & 11.42 & 0.68 & 6.31 \\
\hline 80 & 53986 & 5384 & 48602 & 0.34063 & 0.31267 & 0.02796 & 8.21 & 7.87 & 8.55 & 0.69 & 8.71 \\
\hline 85 & 38495 & 4245 & 34250 & 0.50408 & 0.46421 & 0.03988 & 7.91 & 5.61 & 6.31 & 0.70 & 12.45 \\
\hline 90 & 22888 & 2746 & 20142 & 0.71110 & 0.66469 & 0.04641 & 6.53 & 3.81 & 4.61 & 0.80 & 21.03 \\
\hline 95 & 7469 & 934 & 6535 & 0.80946 & 0.76557 & 0.04390 & 5.42 & 2.95 & 3.79 & 0.84 & 28.36 \\
\hline 100 & 765 & 106 & 659 & 0.93420 & 0.90407 & 0.03013 & 3.23 & 2.04 & 2.99 & 0.96 & 46.92 \\
\hline
\end{tabular}


TABELLA 6 - Tavole di mortalità in presenza ed in assenza della causa di morte: "Malattie del sistema respiratorio" - Italia - Femmine - 2007.

\begin{tabular}{|c|c|c|c|c|c|c|c|c|c|c|c|}
\hline \multirow[b]{2}{*}{ Età $\mathrm{x}$} & \multicolumn{3}{|c|}{ Decessi osservati } & \multicolumn{4}{|c|}{ Quozienti di mortalità } & \multicolumn{4}{|c|}{ Speranza di vita } \\
\hline & ${ }_{n} D_{x}$ & ${ }_{n} D_{x, i}$ & ${ }_{n} D_{x, \neq i}$ & $n q_{x}$ & $n q_{x, \neq i}$ & Diff. ass. & Diff. \% & $\mathbf{e}_{\mathrm{x}}$ & $e_{x, \neq i}$ & Diff. ass. & Diff. $\%$ \\
\hline A & B & C & $D=B-C$ & $\mathbf{E}$ & $\mathbf{F}$ & $G=E-F$ & $H=100^{*} G / E$ & 1 & $\mathbf{L}$ & $M=L-I$ & $\mathrm{~N}=100 * \mathrm{M} / \mathrm{I}$ \\
\hline 0 & 889 & 14 & 875 & 0.00314 & 0.00309 & 0.00005 & 1.57 & 84.04 & 84.48 & 0.43 & 0.52 \\
\hline 1 & 164 & 6 & 158 & 0.00055 & 0.00053 & 0.00002 & 3.66 & 83.31 & 83.74 & 0.43 & 0.52 \\
\hline 5 & 113 & 4 & 109 & 0.00041 & 0.00040 & 0.00001 & 3.54 & 79.35 & 79.78 & 0.43 & 0.54 \\
\hline 10 & 139 & 4 & 135 & 0.00046 & 0.00045 & 0.00001 & 2.88 & 74.38 & 74.81 & 0.43 & 0.58 \\
\hline 15 & 299 & 10 & 289 & 0.00086 & 0.00084 & 0.00003 & 3.34 & 69.42 & 69.84 & 0.43 & 0.62 \\
\hline 20 & 316 & 8 & 308 & 0.00102 & 0.00099 & 0.00003 & 2.53 & 64.47 & 64.90 & 0.43 & 0.66 \\
\hline 25 & 423 & 13 & 410 & 0.00114 & 0.00110 & 0.00003 & 3.07 & 59.54 & 59.96 & 0.43 & 0.71 \\
\hline 30 & 656 & 20 & 636 & 0.00152 & 0.00148 & 0.00005 & 3.05 & 54.60 & 55.03 & 0.42 & 0.78 \\
\hline 35 & 1155 & 21 & 1134 & 0.00238 & 0.00234 & 0.00004 & 1.82 & 49.68 & 50.10 & 0.42 & 0.85 \\
\hline 40 & 1970 & 44 & 1926 & 0.00398 & 0.00389 & 0.00009 & 2.23 & 44.79 & 45.21 & 0.42 & 0.94 \\
\hline 45 & 2864 & 59 & 2805 & 0.00633 & 0.00620 & 0.00013 & 2.05 & 39.96 & 40.38 & 0.42 & 1.05 \\
\hline 50 & 4066 & 77 & 3989 & 0.01016 & 0.00997 & 0.00019 & 1.88 & 35.20 & 35.62 & 0.42 & 1.19 \\
\hline 55 & 6142 & 147 & 5995 & 0.01573 & 0.01535 & 0.00037 & 2.37 & 30.53 & 30.95 & 0.42 & 1.37 \\
\hline 60 & 8628 & 212 & 8416 & 0.02450 & 0.02390 & 0.00059 & 2.43 & 25.98 & 26.39 & 0.42 & 1.61 \\
\hline 65 & 13873 & 434 & 13439 & 0.03876 & 0.03757 & 0.00119 & 3.07 & 21.56 & 21.98 & 0.42 & 1.94 \\
\hline 70 & 20980 & 851 & 20129 & 0.06465 & 0.06211 & 0.00254 & 3.93 & 17.32 & 17.74 & 0.42 & 2.40 \\
\hline 75 & 36275 & 1812 & 34463 & 0.11945 & 0.11384 & 0.00561 & 4.70 & 13.33 & 13.75 & 0.42 & 3.12 \\
\hline 80 & 57518 & 3296 & 54222 & 0.22631 & 0.21485 & 0.01146 & 5.06 & 9.77 & 10.19 & 0.42 & 4.30 \\
\hline 85 & 58471 & 3571 & 54900 & 0.38820 & 0.36956 & 0.01864 & 4.80 & 6.85 & 7.30 & 0.45 & 6.59 \\
\hline 90 & 49734 & 3559 & 46175 & 0.61885 & 0.59161 & 0.02724 & 4.40 & 4.56 & 5.11 & 0.56 & 12.27 \\
\hline 95 & 23434 & 1888 & 21546 & 0.77428 & 0.74552 & 0.02876 & 3.71 & 3.23 & 3.90 & 0.67 & 20.88 \\
\hline 100 & 3921 & 373 & 3548 & 0.92195 & 0.90052 & 0.02143 & 2.32 & 2.15 & 3.01 & 0.86 & 39.90 \\
\hline
\end{tabular}


TABELLA 7 - Tavole di mortalità in presenza ed in assenza della causa di morte: "Cause esterne di traumatismo ed avvelenamento" - Italia - Maschi - 2007.

\begin{tabular}{|c|c|c|c|c|c|c|c|c|c|c|c|}
\hline \multirow[b]{2}{*}{ Età $x$} & \multicolumn{3}{|c|}{ Decessi osservati } & \multicolumn{4}{|c|}{ Quozienti di mortalità } & \multicolumn{4}{|c|}{ Speranza di vita } \\
\hline & ${ }_{n} D_{x}$ & ${ }_{n} D_{x, i}$ & ${ }_{n} D_{x, \neq i}$ & $n q_{x}$ & $n q_{x, \neq i}$ & Diff. ass. & Diff. \% & $\mathbf{e}_{\mathrm{x}}$ & $\mathbf{e}_{x, \neq i}$ & Diff. ass. & Diff. \% \\
\hline A & B & C & $D=B-C$ & E & $\mathbf{F}$ & $G=E-F$ & $H=100^{\star} G / E$ & I & L & $M=L-I$ & $\mathrm{~N}=100^{\star} \mathrm{M} / \mathrm{I}$ \\
\hline 0 & 1070 & 17 & 1053 & 0.00379 & 0.00373 & 0.00006 & 1.59 & 78.67 & 79.69 & 1.02 & 1.30 \\
\hline 1 & 197 & 43 & 154 & 0.00070 & 0.00055 & 0.00015 & 21.84 & 77.97 & 78.99 & 1.02 & 1.31 \\
\hline 5 & 153 & 38 & 115 & 0.00050 & 0.00038 & 0.00012 & 24.86 & 74.02 & 75.03 & 1.01 & 1.36 \\
\hline 10 & 198 & 80 & 118 & 0.00067 & 0.00040 & 0.00027 & 40.43 & 69.06 & 70.06 & 1.00 & 1.45 \\
\hline 15 & 759 & 522 & 237 & 0.00227 & 0.00071 & 0.00156 & 68.73 & 64.10 & 65.08 & 0.98 & 1.53 \\
\hline 20 & 1153 & 802 & 351 & 0.00343 & 0.00104 & 0.00238 & 69.52 & 59.24 & 60.13 & 0.89 & 1.50 \\
\hline 25 & 1473 & 923 & 550 & 0.00378 & 0.00141 & 0.00237 & 62.62 & 54.44 & 55.19 & 0.75 & 1.38 \\
\hline 30 & 1736 & 926 & 809 & 0.00383 & 0.00179 & 0.00204 & 53.33 & 49.63 & 50.26 & 0.63 & 1.27 \\
\hline 35 & 2469 & 904 & 1564 & 0.00482 & 0.00306 & 0.00176 & 36.58 & 44.82 & 45.35 & 0.53 & 1.19 \\
\hline 40 & 3629 & 890 & 2738 & 0.00714 & 0.00539 & 0.00175 & 24.47 & 40.02 & 40.48 & 0.46 & 1.15 \\
\hline 45 & 4995 & 838 & 4157 & 0.01110 & 0.00925 & 0.00185 & 16.70 & 35.29 & 35.69 & 0.40 & 1.13 \\
\hline 50 & 6988 & 708 & 6280 & 0.01803 & 0.01622 & 0.00181 & 10.05 & 30.65 & 31.00 & 0.34 & 1.12 \\
\hline 55 & 11075 & 720 & 10355 & 0.02995 & 0.02803 & 0.00192 & 6.41 & 26.17 & 26.47 & 0.30 & 1.14 \\
\hline 60 & 16021 & 675 & 15346 & 0.04818 & 0.04620 & 0.00198 & 4.11 & 21.89 & 22.16 & 0.26 & 1.21 \\
\hline 65 & 24320 & 777 & 23543 & 0.07582 & 0.07348 & 0.00233 & 3.07 & 17.87 & 18.11 & 0.25 & 1.37 \\
\hline 70 & 33993 & 940 & 33053 & 0.12462 & 0.12139 & 0.00323 & 2.59 & 14.12 & 14.35 & 0.23 & 1.65 \\
\hline 75 & 47018 & 1236 & 45782 & 0.20759 & 0.20273 & 0.00486 & 2.34 & 10.74 & 10.99 & 0.24 & 2.25 \\
\hline 80 & 53986 & 1460 & 52526 & 0.34063 & 0.33316 & 0.00747 & 2.19 & 7.87 & 8.14 & 0.27 & 3.48 \\
\hline 85 & 38495 & 1219 & 37277 & 0.50408 & 0.49295 & 0.01113 & 2.21 & 5.61 & 5.96 & 0.35 & 6.28 \\
\hline 90 & 22888 & 729 & 22159 & 0.71110 & 0.69945 & 0.01166 & 1.64 & 3.81 & 4.33 & 0.52 & 13.67 \\
\hline 95 & 7469 & 266 & 7203 & 0.80946 & 0.79785 & 0.01161 & 1.43 & 2.95 & 3.58 & 0.63 & 21.41 \\
\hline 100 & 765 & 17 & 748 & 0.93420 & 0.93009 & 0.00411 & 0.44 & 2.04 & 2.86 & 0.82 & 40.18 \\
\hline
\end{tabular}


TABELLA 8 - Tavole di mortalità in presenza ed in assenza della causa di morte: "Cause esterne di traumatismo ed avvelenamento" - Italia - Femmine - 2007.

\begin{tabular}{|c|c|c|c|c|c|c|c|c|c|c|c|}
\hline \multirow[b]{2}{*}{ Età $x$} & \multicolumn{3}{|c|}{ Decessi osservati } & \multicolumn{4}{|c|}{ Quozienti di mortalità } & \multicolumn{4}{|c|}{ Speranza di vita } \\
\hline & ${ }_{n} D_{x}$ & ${ }_{n} D_{x, i}$ & ${ }_{n} D_{x, \neq i}$ & $n q_{x}$ & $n q_{x, \neq i}$ & Diff. ass. & Diff. \% & $\mathbf{e}_{\mathrm{x}}$ & $\mathbf{e}_{x, \neq i}$ & Diff. ass. & Diff. \% \\
\hline A & B & C & $D=B-C$ & E & $\mathbf{F}$ & $G=E-F$ & $H=100^{*} G / E$ & $\mathbf{I}$ & $\mathbf{L}$ & $M=L-I$ & $\mathrm{~N}=100^{\star} \mathrm{M} / \mathrm{I}$ \\
\hline 0 & 889 & 12 & 877 & 0.00314 & 0.00310 & 0.00004 & 1.35 & 84.04 & 84.45 & 0.41 & 0.49 \\
\hline 1 & 164 & 26 & 138 & 0.00055 & 0.00046 & 0.00009 & 15.85 & 83.31 & 83.72 & 0.41 & 0.49 \\
\hline 5 & 113 & 20 & 93 & 0.00041 & 0.00034 & 0.00007 & 17.70 & 79.35 & 79.75 & 0.40 & 0.51 \\
\hline 10 & 139 & 32 & 107 & 0.00046 & 0.00036 & 0.00011 & 23.02 & 74.38 & 74.78 & 0.40 & 0.53 \\
\hline 15 & 299 & 152 & 147 & 0.00086 & 0.00042 & 0.00044 & 50.83 & 69.42 & 69.81 & 0.39 & 0.56 \\
\hline 20 & 316 & 144 & 172 & 0.00102 & 0.00055 & 0.00046 & 45.56 & 64.47 & 64.83 & 0.36 & 0.56 \\
\hline 25 & 423 & 141 & 282 & 0.00114 & 0.00076 & 0.00038 & 33.32 & 59.54 & 59.87 & 0.33 & 0.56 \\
\hline 30 & 656 & 153 & 503 & 0.00152 & 0.00117 & 0.00035 & 23.31 & 54.60 & 54.91 & 0.31 & 0.57 \\
\hline 35 & 1155 & 170 & 985 & 0.00238 & 0.00203 & 0.00035 & 14.70 & 49.68 & 49.97 & 0.29 & 0.59 \\
\hline 40 & 1970 & 227 & 1743 & 0.00398 & 0.00352 & 0.00046 & 11.50 & 44.79 & 45.07 & 0.28 & 0.62 \\
\hline 45 & 2864 & 195 & 2669 & 0.00633 & 0.00590 & 0.00043 & 6.79 & 39.96 & 40.22 & 0.26 & 0.65 \\
\hline 50 & 4066 & 195 & 3871 & 0.01016 & 0.00967 & 0.00048 & 4.77 & 35.20 & 35.44 & 0.25 & 0.70 \\
\hline 55 & 6142 & 166 & 5976 & 0.01573 & 0.01530 & 0.00042 & 2.68 & 30.53 & 30.77 & 0.23 & 0.77 \\
\hline 60 & 8628 & 247 & 8381 & 0.02450 & 0.02380 & 0.00069 & 2.83 & 25.98 & 26.21 & 0.23 & 0.88 \\
\hline 65 & 13873 & 328 & 13545 & 0.03876 & 0.03786 & 0.00090 & 2.32 & 21.56 & 21.78 & 0.22 & 1.03 \\
\hline 70 & 20980 & 471 & 20509 & 0.06465 & 0.06325 & 0.00140 & 2.17 & 17.32 & 17.54 & 0.22 & 1.26 \\
\hline 75 & 36275 & 843 & 35432 & 0.11945 & 0.11685 & 0.00261 & 2.18 & 13.33 & 13.56 & 0.22 & 1.68 \\
\hline 80 & 57518 & 1701 & 55817 & 0.22631 & 0.22042 & 0.00589 & 2.60 & 9.77 & 10.02 & 0.25 & 2.52 \\
\hline 85 & 58471 & 1924 & 56547 & 0.38820 & 0.37823 & 0.00997 & 2.57 & 6.85 & 7.15 & 0.30 & 4.37 \\
\hline 90 & 49734 & 1656 & 48078 & 0.61885 & 0.60641 & 0.01244 & 2.01 & 4.56 & 4.98 & 0.42 & 9.22 \\
\hline 95 & 23434 & 802 & 22632 & 0.77428 & 0.76248 & 0.01180 & 1.52 & 3.23 & 3.79 & 0.56 & 17.40 \\
\hline 100 & 3921 & 122 & 3799 & 0.92195 & 0.91550 & 0.00645 & 0.70 & 2.15 & 2.93 & 0.78 & 36.22 \\
\hline
\end{tabular}




\section{BIBLIOGRAFIA}

Adamic, P.; Dixon, S.; Gillis, D.; (2010). "Multiple decrement modelling in the presence of interval censoring and masking”. Scandinavian Actuarial Journal, Volume 2010, Issue 4.

Alho, J.M.; Saari, M.; Juolevi, A.; (2000). "A competing risks approach to the two-sex problem”. Mathematical Population Studies, Volume 8, Issue 1.

Arthur, W.B.; Stoto, M.A. (1983). “An analysis of indirect mortality estimation”, Population Studies, 37.

Benjamin, B.; Pollard, J.H.; (1970). “The Analysis of Mortality and Other Actuarial Statistics". Heinemann, London.

Boulier, B.L.; Paqueo, V.B. (1988). "On the measurement of the determinants of mortality”. Demography, 25(2).

Caselli, G.; Egidi. V. (1981). "Le differenze territoriali di mortalità in Italia. Tavole di mortalità provinciali (1971-72)”. Roma, Istituto di Demografia dell'Università 'La Sapienza'.

Chiang, C.L. ; (1968). "Competing Risks". Introduction to Stochastic Processes in Biostatistics. John Wiley \& Sons, New York.

Chiang, C.L. ; (1991). “Competing Risks in mortality analysis”. Annual Review of Public Health., Volume12.

Colvez, A.; Blanchet, M.; (1983). "Potential Gains in Life Expectancy Free of Disability; A Tool for Health Planning”. International Journal of Epidemiology, Volume 12 , Issue 2.

Conti, S.; Farchi, G.; Masocco, M.; Toccaceli, V.; Vichi, M.; (1999). "The Impact of the Major Causes of Death on Life Expectancy in Italy”. International Journal of Epidemiology, Volume 28, Issue 5.

Courgeau, D.; Lelievre, E.; (1994). "Competing risks and independence: a theoretical framework for reflection”. Population, Vol. 49, No. 2.

Ebrahimi, N.; (1996). "The effects of misclassification of the actual cause of death in competing risk analysis”. Statistics in Medicine, Vol. 15, No. 14.

Geronimus, A.T.; Bound, J.; Waidmann, T.A.; Colen C.G.; Steffick, D.; (2001). "Inequality in Life Expectancy, Functional Status, and Active Life Expectancy across Selected Black and White Populations in the United States”. Demography, Vol. 38, No. 2. 
Halli S.S.; Rao K.V.; (1992). "Advanced Techniques of Populations Analysis". Plenum Press, New York.

Hinde, A.; (1998). "Demographic Methods". Arnold, London.

Hsieh, J.J.; (1989). "A probabilistic approach to the construction of competing-risk life tables". Biometrical Journal, Vol. 31, No. 3.

ISTAT; (2010). "Cause di Morte - Italia - Anno 2007”. Pubblicazione on line disponibile sul sito Istat.

ISTAT; (2010). “Annuario Statistico Italiano”. R.T.I. Poligrafica Ruggiero, Avellino.

Keyfitz, N.; Caswell H., (2005). "Applied Mathematical Demography". Third Edition. Springer, USA.

Lai D.; Tsai S.P. ; Hardy R.J. ; (1977). "Impact of HIV/AIDS on life expectancy in the United States". AIDS, 11.

Lai D.; Hardy R.J. ; (1999). "Potential gains in life expectancy or years of potential life lost: impact of competing risks of death". International Journal of Epidemiology, 28.

Namboodiri, K.; Suchindran C.M., (1987). "Life Table Techniques and their Applications". Academic Press, London.

Petrioli, L. (1968). "Evoluzione della mortalità per cause in Italia dal 1899 al 1962, con previsioni fino al 1980". Rivista italiana di Economia, Demografia e Statistica - Volume XXII, Fascicolo 1.

Petrioli, L. (1979). "Mortalità per cause: Confronti fra alcune stime e le relative osservazioni”. Quaderni dell'Istituto di Statistica - Siena - Numero 38.

Pollard, J.H.; (1988). "On the Decomposition of Changes in Expectation of Life and Differentials in Life Expectancy”. Demography, Vol. 25.

Preston, S.H.; Heuveline P.; Guillot M.; (2001). "Demography - Measuring and Modeling Populations Processes". Blackwell Publishers, Oxford.

Schmidbauer, H. ; (1992). "Some aspect of competing risks in demography". In: "Economic evolution and demographic change: formal models in social sciences". Springer-Verlag, New York.

Tsai, S.P.; Lee, E.S. ; Hardy, R.J. ; (1978). "The Effect of a Reduction in Leading Causes of Death: Potential Gains in Life Expectancy". AJPH, October 1978, Vol. 68, No. 10.

Vassin, S.;. (1994). 'Epidemiological transition of mortality curves in terms of the Brass logit system'. European Journal of Population, 10. 


\section{QUADERNI DEL DIPARTIMENTO DI STUDI AZIENDALI E SOCIALI SEZIONE: "POPOLAZIONE, AMBIENTE E METODI MATEMATICO-STATISTICI" FACOLTÀ DI ECONOMIA "R. M. GOODWIN"}

1) Petrioli Luciano - "Relational methods in demography" - Siena, novembre 1996.

2) Petrioli Luciano - "A new female fertility function" - Siena, dicembre 1996.

3) Fineschi Franco, Giannetti Riccardo e Lusini Silvia - "A new algorithm for least squares solutions" - Siena, gennaio 1997.

4) Petrioli Luciano - "Complete and truncated distributions of fertility rates" Siena, aprile 1997.

5) Salerno Tiziano - "Un modello di previsione della domanda di ricovero ospedaliero da parte della popolazione senese (anni 1999-2001)" - Siena, dicembre 1999.

6) Buccianti Cinzia - "La demografia di due realtà toscane alla fine del secondo millennio" - Siena, 2001.

7) Buccianti Cinzia - "La distribuzione della popolazione" - Siena, luglio 2002.

8) Buccianti Cinzia - "Il fenomeno migratorio ad Avezzano: uno studio della fonte anagrafica" - Siena, luglio 2002.

9) Menchiari Andrea - "Un nuovo metodo relazionale per la proiezione delle probabilità di morte - Il caso dell'Italia (2005-2050)" - Siena, marzo 2007.

10) Fineschi Franco, Fontani Sandra - "Geographic decentralization of facilities and service systems: an ad hoc approach" - Siena, settembre 2007.

11) Fontani Sandra - "Profit maximizing location of facilities and service systems" - Siena, settembre 2007. 